\title{
Sensitivity of Calibration to Principal Point Position
}

\author{
R.I. Hartley ${ }^{1}$ and R. Kaucic ${ }^{2}$ \\ 1 Dept. of Systems Eng., RSISE, A.N.U., ACT, 0200 Australia \\ Richard.Hartley@anu.edu.au \\ 2 G.E.-CRD, Schenectady, New Yorck, 12309 U.S.A. \\ kaucic@crd.ge.com
}

\begin{abstract}
A common practice when carrying out self-calibration and Euclidean reconstruction from one or more views is to start with a guess at the principal point of the camera. The general belief is that inaccuracies in the estimation of the principal point do not have a significant effect on the other calibration parameters, or on reconstruction accuracy. It is the purpose of this paper to refute that belief. Indeed, it is demonstrated that the determination of the focal length of the camera is tied up very closely with the estimate of the principal point. Small changes in the estimated (sometimes merely guessed) principal point can cause very large changes in the estimated focal length, and the accuracy of reconstruction. In fact, the relative uncertainty in the focal length is inversely proportional to the distance of the principal point to the epipolar line. This analysis is geometric and exact, rather than experimental.
\end{abstract}

\section{Introduction}

The principal point of a camera is often quite difficult to determine, and is known often to vary with the zoom setting of a camera. Often, therefore, the principal point is guessed when calibration or self-calibration of the camera takes place ([2.10]). The generally held opinion is that this makes little difference to the estimate of the other camera parameters, or to the ultimate accuracy of Euclidean reconstruction. It is possible that this may be true under some conditions, but it has been quoted as a general principal often without critical examination. The purpose of this paper is to point out that this assumption is far from safe, and that in fact the estimated position of the principal point may have a large effect on the accuracy of calibration and reconstruction.

We are concerned throughout with natural pinhole cameras, that is cameras with zero skew and unit (or known) aspect ratio. First, we consider calibration of a camera from a single view, in which a horizon line and a vertical direction, or vertical vanishing point may be identified. Given knowledge of the principal point, the focal length may be determined by an easy geometric construction, from which the dependence of the focal length on the principal point estimate is easily seen.

This single-view case is next extended to the two-view case, in which the focal lengths of each camera may be determined from the fundamental matrix alone. It is shown that this problem may be reduced to the previous case of single-view calibration, by considering the horizon line and perpendicular vanishing direction of the plane formed by the base line and one of the camera's principal rays. The sensitivity of this process to variations in the principal point then becomes evident. Estimation of the focal length in 
this manner is well known to be unstable ([י2). In part this is due to sensitivity in the estimation of the fundamental matrix of the camera pair. It is shown here, however, that inaccuracy in the assumed position of the focal length is also a major culprit.

In an influential paper ([2]) Bougnoux gives a beautifully simple formula for the focal length of the camera estimated from two views. As noted above, he finds that the estimate of the focal length is quite sensitive, which we confirm. However he reiterates the popular belief that the assumed position of the principal point does not have an important effect on the estimated focal length, or on the accuracy of an eventual projective reconstruction of the scene. This view has also been stated (often without sufficient evidence) by other researchers, but is contrary to the findings of the present investigation. Indeed one of the purposes of this paper is to demonstrate that these two findings are not universally true, and must be treated with caution. By giving a new geometrically intuitive interpretation of certain one and two-view self-calibration methods, we provide the user with a tool that can be used to ascertain whether the assumptions are valid in a particular reconstruction scenario.

\section{Calibration from a Single Image}

Throughout this paper, we assume that the skew of the camera is zero, and the aspect ratio is equal to one. Thus, the only remaining parameters of the camera are the focal length and the principal point.

We consider first calibration of the camera (determination of the focal length and principal point) from a single view. Clearly this can not be done without some scene information, and many ways have been proposed to do this. For instance, if three orthogonal vanishing points can be identified in the image, then the principal point may be identified as the orthocentre of the triangle formed by the three vanishing points ([3]). In addition, the focal length may be determined by direct geometric construction ([8], page 212, [4]).

Thus, if the vanishing points of all three orthogonal directions are known, then the camera may be completely calibrated without any further assumptions. Therefore, we consider a calibration problem in which there is a little less information available. Accordingly, we assume that the horizon line and the vertical vanishing point (henceforth known as the apex) are identified in an image. An example of such an image is given in Fig. 1. The principal point in the image must lie on the perpendicular from the apex to the horizon. Thus, the principal point has a single remaining degree of freedom. Suppose that the principal point is the point $\mathbf{p}$ in the image, $\mathbf{v}$ is the vertex, and $\mathbf{h}$ is the foot of the perpendicular from the apex to the horizon. In this case, the focal length may be computed by a simple formula:

$$
f^{2}=-d(\mathbf{p}, \mathbf{h}) d(\mathbf{p}, \mathbf{v})
$$

where $f$ is the focal length, and $d(\cdot, \cdot)$ represents the (signed) Euclidean distance. The negative sign in this expression expresses the fact that $d(\cdot, \cdot)$ is a signed quantity, and that the direction vectors from the principal point to the horizon and the apex must be in opposite directions in order for $f^{2}$ to be positive, as given by this formula. In other words, the principal point must lie between the horizon and the apex. Proof of this formula is given in Fig. 2. 

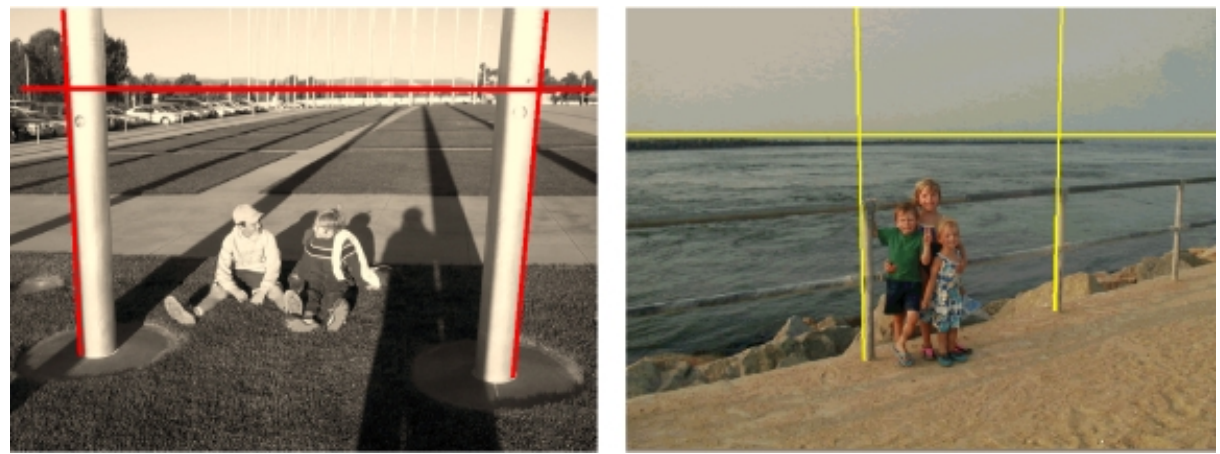

Fig. 1. Examples of images for which it is possible to compute the focal length of the camera, assuming knowledge of the principal point. To do this, it is sufficient to identify the horizon and a vertical vanishing direction. In the left-hand image, the exact position of the horizon can be established with the aid of the vanishing points of the ground markings and shadows. The image of the apex may be computed as the intersection of the the two vertical edges. Focal length may then be computed using (1).
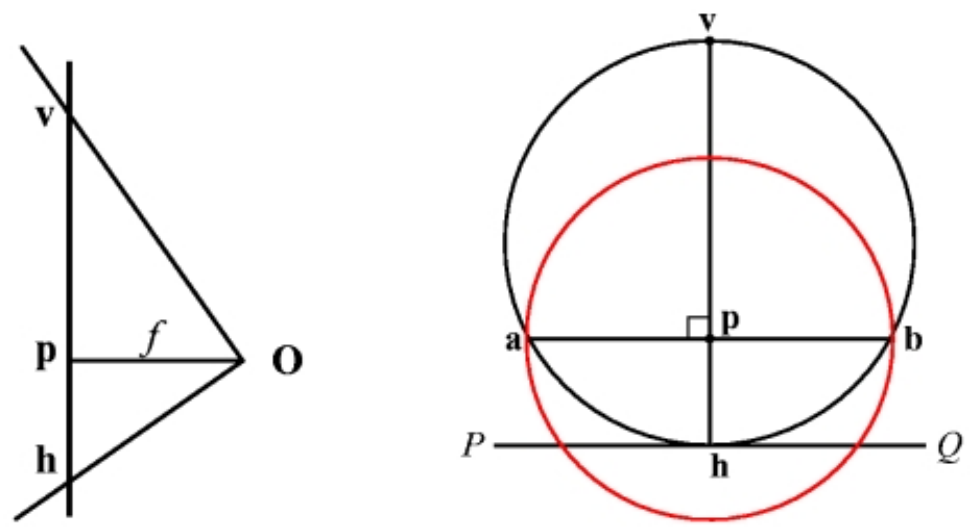

Fig. 2. Left. The diagram shows the vertical plane containing the principal ray $p O$ of the camera. The two rays to the horizon and the apex are perpendicular to each other, meeting the focal plane at points $\mathbf{h}$ and $\mathbf{v}$ respectively. The focal length is the distance from the camera centre to the focal plane. By similar triangles, $f^{2}=-d(\mathbf{p}, \mathbf{h}) d(\mathbf{p}, \mathbf{v})$.

Right. In the image, a circle (black) is drawn with diameter the perpendicular line between the apex $\mathbf{v}$ and the horizon $P Q$. The principal point must lie on this line. A line is drawn through $\mathbf{p}$ perpendicular to $\mathbf{v h}$. This meets the circle in two points $\mathbf{a}$ and $\mathbf{b}$. The focal length equals the distance $d(\mathbf{p}, \mathbf{a})$, and the circle with diameter $\mathbf{a b}$ is the $45^{\circ}$ circle in the image (shown in red).

The focal length may be computed by a simple geometric construction, as follows. Let $C$ be the circle with the line from apex $\mathbf{v}$ to horizon $\mathbf{h}$ as diameter. The line in the image through the principal point $\mathbf{p}$ meets the circle $C$ in two points a and $\mathbf{b}$. By elementary geometry of a circle, $d(\mathbf{p}, \mathbf{a}) d(\mathbf{p}, \mathbf{b})=d(\mathbf{p}, \mathbf{v}) d(\mathbf{p}, \mathbf{h})$, and since $d(\mathbf{p}, \mathbf{a})=-d(\mathbf{p}, \mathbf{b})$ 
it follows that $f=d(\mathbf{p}, \mathbf{a})$. The circle constructed with centre $\mathbf{p}$ passing through $\mathbf{a}$ and b is the $45^{\circ}$ circle in the image. This is illustrated in Fig. 2, and on a real image in Fig. 3. Since $f$ is the distance from $\mathbf{p}$ to the circle $C$ along a line drawn perpendicular to the diameter vh, the way the estimated value of $f$ varies as a function of the presumed principal point location is easily visualized. In particular, as the assumed principal point moves towards the horizon, or the apex in the image, the corresponding estimated value of the focal length $f$ diminishes towards zero. If an image is taken with a camera aimed directly at the horizon, the principal point corresponds with the point $\mathbf{h}$ on the horizon, and so $d(\mathbf{p}, \mathbf{h})=0$. In this case, however, the distance to the apex will be infinite, and hence $f^{2}=0 \times \infty$, and the computed value of $f$ will be indeterminate. From this, we see that it is impossible to compute $f$ from this configuration if the camera is pointed directly at the horizon, or the apex.

\subsection{A Different Formula}

It is sometimes difficult to measure the distance of the principal point to the apex, particularly if the apex is far away from the visible area of the image. A simple modification of the image takes care of that problem. Suppose that $l$ is a line from the apex to the horizon, not passing through the principal point. This can be the image of a vertical object in the scene. Let $s_{1}=d(\mathbf{p}, \mathbf{h})$ be the orthogonal distance from the principal point to the horizon, and let $s_{2}=d(\mathbf{p}, \mathbf{l})$ be the orthogonal distance from the principal point to the line 1 . Let $\theta$ be the angle between line $l$ and the horizon - more specifically, the angle of the region containing the principal point. Then

$$
f^{2}=s_{1} s_{2} / \cos \theta
$$

The proof of this is immediate from Fig. 4. Note that for $f^{2}$ to be positive, the principal point must lie in the acute-angled section of the plane partitioned by lines $h$ and $l$.

Sensitivity of $f$. In terms of (2) it is easy to write down an expression for the uncertainty of the focal length in terms of small errors in the three parameters $s_{1}, s_{2}$ and $\theta$. Since $d f=\partial f / \partial s_{1} d s_{1}+\partial f / \partial s_{2} d s_{2}+\partial f / \partial \theta d \theta$, simple calculus leads to the following formula for the relative change in $f$.

$$
d f / f=1 / 2\left(d s_{1} / s_{1}+d s_{2} / s_{2}+\tan \theta d \theta\right)
$$

From this we see that $f$ is sensitive to small changes in the measurements of $s_{1}, s_{2}$ and $\theta$ when $s_{1}$ or $s_{2}$ are small, or $\theta$ is close to $90^{\circ}$. In particular, it is impossible to estimate $f$ accurately when the principal point is close to the horizon line, since in this case $s_{1}$ is small, and the angle $\theta$ will be close to $90^{\circ}$.

Dependence of $f$ on the principal point. It was seen above that if the horizon and apex are identifiable in an image, then the principal point position may vary with one degree of freedom, along the line through the apex perpendicular to the horizon. If there is only one vertical line feature visible in the image, then the apex may not be identified, but instead may lie anywhere along the image of the vertical line. The principal point may now vary with two degrees of freedom anywhere in the image. Figure 5 gives an example of such an image. For a choice of the principal point, the focal length may be computed 

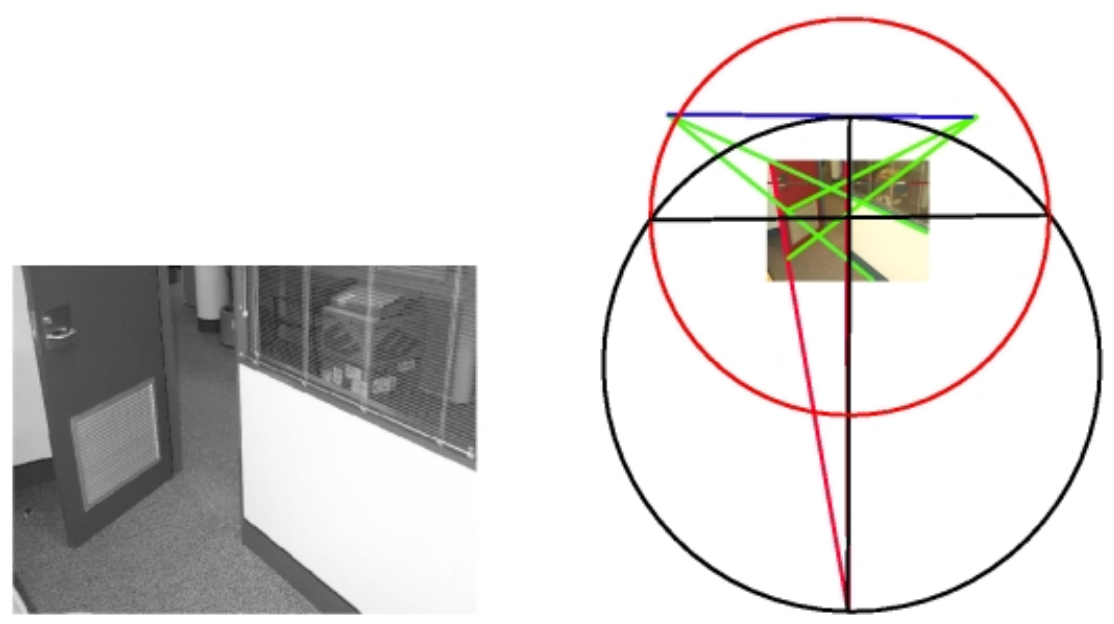

Fig. 3. This image shows the computation of the focal length of the camera assuming a principal point at the centre of the image. The original image is shown to the left, and the construction to the right. Since the horizon line (blue) is not directly visible in this image, it is computed as the line joining two horizontal vanishing points, computed from the right-hand wall and the grid on the door (green lines). Note that these are not orthogonal vanishing points. The vertical vanishing point is given by the sides of the door and the door frame (red lines). The red circle is the $45^{\circ}$ circle, with radius $f$, assuming the principal point is placed as shown (at the centre of the red circle).

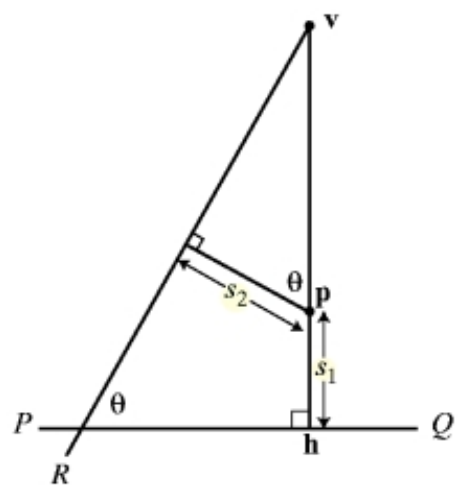

Fig. 4. Since $d(\mathbf{p}, \mathbf{v})=s_{2} / \cos \theta$, it follows from (1) that $f^{2}=s_{1} s_{2} / \cos \theta$.

using (2), or by direct geometric construction, as shown previously. However, because of the added degree of freedom, the computed focal length depends more dramatically on the assumed position of the principal point. It may vary from near zero, when the principal point is assumed to be close to the intersection of the horizon and vertical line, to large values when the principal point is far from both these lines. 


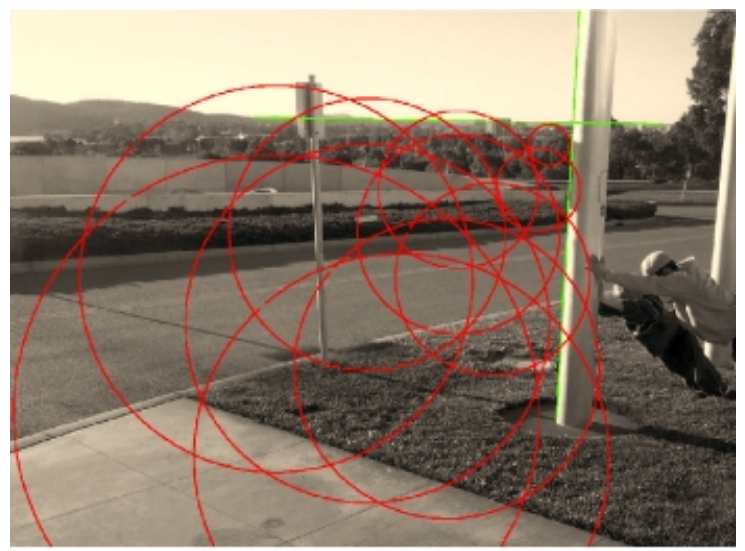

Fig. 5. In this image, the apex is unknown, but is constrained to lie somewhere along the vertical direction shown in the image (green). The principal point has two degrees of freedom, but once a position of the principal point is assumed, the focal length of the camera may be computed. The red circles represent various estimates of the focal length for different assumed principal points. Each circle is the locus of all directions $10^{\circ}$ from the principal point, which is at the centre of the circle. Thus, the radius of each circle is equal to $0.176 f=\tan \left(10^{\circ}\right) f$. The focal length has been computed in each case using (2).

\section{Calibration from Two Views}

Next, the calibration methods for a single view discussed above will be extended to consideration of calibration from two views. This problem was first addressed in [5] where an algorithm for computing the two focal lengths was given. This algorithm was quite complicated, and the problem was later considered by several authors. The most compact solution was found by Bougnoux in [2], where a simple formula was given for the two focal lengths in terms of the fundamental matrix and the two principal points (see equation (5) below). This formula will be rederived here in a way that sheds light on its geometric meaning, and its sensitivity to variations in the assumed positions of the principal point. It will be shown that Bougnoux's formula is closely related to (2), and has a simple geometric interpretation.

First, we note that in order to compute the focal lengths, the positions of the two principal points need to be known. The geometry of two views may be summarized in Fig. 6. The two camera centres $O_{1}$ and $O_{2}$ are joined by a baseline $O_{1} O_{2}$ and the view direction of each camera is defined by the principal rays, $R_{1}$ and $R_{2}$ of the cameras. Of most interest is the case where these three lines are not coplanar; in other words, the principal rays of the two cameras do not meet. The principal ray $R_{1}$ and the baseline $\mathrm{O}_{1} \mathrm{O}_{2}$ define a plane, which will be denoted by $\mathrm{R}_{1} O_{1} O_{2}$. In addition, let $A_{1}$ be the ray through the camera centre $O_{1}$ perpendicular to the plane $\mathrm{R}_{1} \mathrm{O}_{1} \mathrm{O}_{2}$. This plane, and the ray $A_{1}$ form an orthogonal pair in space. If their projections into the second image can be identified, then we may use the methods of section[2 to compute the focal length of the second camera. 


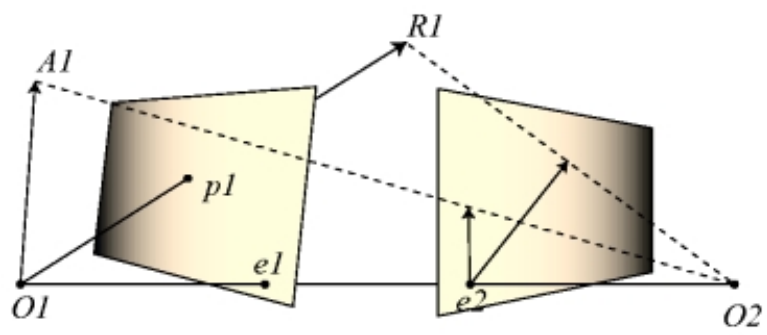

Fig. 6. The plane defined by the principal ray $R_{1}$ and the base-line $\mathrm{O}_{1} \mathrm{O}_{2}$ is imaged as an epipolar line in the second (right-hand) image. The ray $A_{1}$ is perpendicular to this plane, and also projects to an epipolar line in the second image. The two epipolar lines are the images of an orthogonal plane/line pair. Assuming a position for the principal point $\mathbf{p}_{2}$, the focal length $f_{2}$ of the second camera may be computed using the method of section 2 .

First of all, consider the first (left) image taken from camera centre $O_{1}$. We want to identify the projection in this image of the plane $R_{1} O_{1} O_{2}$ and the ray $A_{1}$. The projection of the plane is easily identified as the epipolar line through the principal point, namely $\left[\mathbf{e}_{1}\right]_{\times} \mathbf{p}_{1}=\mathbf{e}_{1} \times \mathbf{p}_{1}$. Since the ray $A_{1}$ is perpendicular to the plane containing the principal ray, it projects to a point at infinity in the image, in the direction perpendicular to the line $\left[\mathbf{e}_{1}\right]_{\times} \mathbf{p}_{1}$. This infinite point may be written as $\mathrm{I}\left(\mathbf{e}_{1} \times \mathbf{p}_{1}\right)$, or $\mathrm{I}\left[\mathbf{e}_{1}\right]_{\times} \mathbf{p}_{1}$, where $\mathrm{I}$ is the $3 \times 3$ matrix $\operatorname{diag}(1,1,0)$.

To explain this last point more fully, note that Il represents the point at infinity in the direction perpendicular to $\mathbf{l}$ for any line $\mathbf{l}$. For example, $(a, b)$ is the vector perpendicular to the line $a x+b y+c=0$, represented by coordinates $(a, b, c)^{\top}$, and $(a, b, 0)$ is the vanishing point in this direction. This relation will be used often in this paper, so we emphasize it:

\section{Proposition 3.1.}

1. If $\mathbf{l}$ is a line, then $\mathrm{Il}$ represents the point at infinity in the direction perpendicular to the line.

2. If line $\mathbf{l}$ passes through point $\mathbf{e}$, then $[\mathbf{e}]_{\times} \mathrm{Il}$ is the line through $\mathbf{e}$ perpendicular to $\mathbf{l}$.

It is now easy to compute the epipolar lines in the second image corresponding to the plane $R_{1} O_{1} O_{2}$ and the ray $A_{1}$. This is done simply by transferring the epipolar lines from the first image to the second, using the fundamental matrix. First, consider the vanishing line of the plane $\mathrm{R}_{1} \mathrm{O}_{1} \mathrm{O}_{2}$. Since this plane passes through the camera centre $\mathrm{O}_{2}$, it is viewed edge-on in the second image. Consequently, its vanishing line is nothing more than the image of any line lying in the plane $\mathrm{R}_{1} \mathrm{O}_{1} \mathrm{O}_{2}$. Since the principal ray of the first camera is just such a line we see:

3.2. The vanishing line in the second image of the plane $R_{1} O_{1} O_{2}$ is the epipolar line $\mathrm{Fp}_{1}$ corresponding to an epipolar line $\left[\mathbf{e}_{1}\right]_{\times} \mathbf{p}_{1}$ in the first image. Here $\mathrm{F}$ is the fundamental matrix.

Secondly, the image of the ray $A_{1}$ is easily computed by transferring its vanishing point using the fundamental matrix: 
3.3. The image of the ray $A_{1}$ in the second image is the epipolar line $\mathrm{FI}\left[\mathbf{e}_{1}\right]_{\times} \mathbf{p}_{1}$. This corresponds to the epipolar line in the first image, perpendicular to $\left[\mathbf{e}_{1}\right]_{\times} \mathbf{p}_{1}$.

Thus, we may conclude:

3.4. Given the fundamental matrix $F$ for two images, and assumed positions of the principal points $\mathbf{p}_{1}$ and $\mathbf{p}_{2}$ in the two images, the focal length $f_{2}$ may be computed as follows.

1. In the first image, the epipolar line through the principal point $\mathbf{p}_{1}$ is given by $\left[\mathbf{e}_{1}\right]_{\times} \mathbf{p}_{1}$. The epipolar line perpendicular to this is $\left[\mathbf{e}_{1}\right]_{\times} \mathbf{I}\left[\mathbf{e}_{1}\right]_{\times} \mathbf{p}_{1}$.

2. The two corresponding epipolar lines in the second image are $F \mathbf{p}_{1}$ and $\mathrm{FI}\left[\mathbf{e}_{1}\right]_{\times} \mathbf{p}_{1}$ respectively.

3. Apply equation (2) where $s_{1}$ and $s_{2}$ are the distances from the principal point $\mathbf{p}_{2}$ to these two lines, and $\theta$ is the angle between them - more specifically, the angle of the segment containing $\mathbf{p}_{2}$.

This construction is illustrated in Fig. (7) An explicit formula is possible. Let $\mathbf{l}$ and $\mathbf{l}^{\prime}$ be the two epipolar lines computed in (3.4). The distance from a point $\mathbf{p}=(x, y, 1)^{\top}$ to a line $\mathbf{l}$ is $\mathbf{l}^{\top} \mathbf{p} / \alpha$ where $\alpha=\left(l_{1}^{2}+l_{2}^{2}\right)^{1 / 2}$, and $l_{i}$ here represents the $i$-th component of the vector $\mathbf{l}$. Furthermore, the angle between two lines $\mathbf{l}$ and $\mathbf{l}^{\prime}$ satisfies the relation $\cos \theta=-\left(\mathbf{l}^{\prime \top} \mathrm{Il}\right) / \alpha \alpha^{\prime}$. Now, writing (2) in terms of the homogeneous coordinates for the two lines $\mathbf{l}$ and $\mathbf{l}^{\prime}$ involved, yields

$$
f^{2}=-\frac{\left(\mathbf{l}^{\top \top} \mathbf{p}\right)\left(\mathbf{l}^{\top} \mathbf{p}\right)}{\mathbf{l}^{\top} \mathrm{Il}^{\prime}}
$$

since the factors $\alpha$ and $\alpha^{\prime}$ cancel top and bottom.

Substituting the formulas (3.2) and (3.3) for the lines $l_{2}$ and $l_{2}^{\prime}$ finally gives the formula

$$
f_{2}^{2}=-\frac{\left(\mathbf{p}_{1}^{\top}\left[\mathbf{e}_{1}\right]_{\times} \mathrm{IF}^{\top} \mathbf{p}_{2}\right)\left(\mathbf{p}_{1}^{\top} \mathrm{F}^{\top} \mathbf{p}_{2}\right)}{\mathbf{p}_{1}^{\top}\left(\left[\mathbf{e}_{1}\right]_{\times} \mathrm{IF}{ }^{\top} \mathrm{IF}\right) \mathbf{p}_{1}} .
$$

In this formula, $\mathbf{e}_{1}$ is the epipole in the first image, and $\mathbf{p}_{1}$ and $\mathbf{p}_{2}$ are homogeneous 3 -vectors representing the principal points in the two images. It is required however, that $\mathbf{p}_{2}=(\cdot, \cdot, 1)^{\top}$, with last coordinate equal to 1 .

The formula for the first focal length $f_{1}$ may be obtained by interchanging the role of the first and second cameras in this formula.

\section{Effect of Varying the Principal Points}

We consider now the effect of varying the principal point in either or both the images. Without loss of generality, let us restrict attention to (5), and computation of $f_{2}^{2}$. Since the left hand side of (5) is the square of the focal length, it is necessary that the right hand side be positive, otherwise, no solution exists. To analyze this further, we note that the expression for $f_{2}^{2}$ may be split into parts as follows:

$$
\begin{aligned}
A\left(\mathbf{p}_{1}, \mathbf{p}_{2}\right) & =\mathbf{p}_{1}^{\top}\left[\mathbf{e}_{1}\right]_{\times} \mathrm{IF}^{\top} \mathbf{p}_{2} \\
B\left(\mathbf{p}_{1}, \mathbf{p}_{2}\right) & =\mathbf{p}_{1}^{\top} \mathrm{F}^{\top} \mathbf{p}_{2} \\
D\left(\mathbf{p}_{1}\right) & =\mathbf{p}_{1}^{\top}\left(\left[\mathbf{e}_{1}\right]_{\times} \mathrm{IF}{ }^{\top} \mathrm{IF}\right) \mathbf{p}_{1}
\end{aligned}
$$



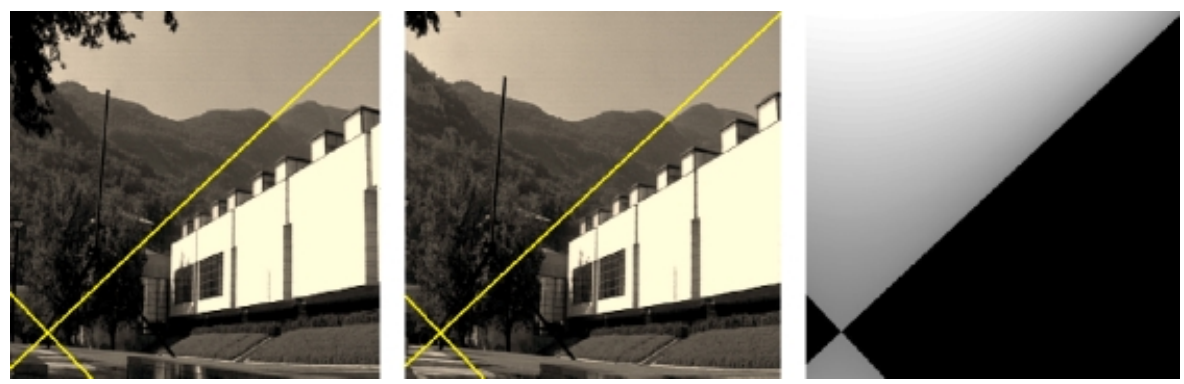

Fig. 7. A pair of images of the Grenoble museum from which one wishes to compute the focal lengths. It is assumed that the principal point $\mathbf{p}_{1}$ in the first image is the centre of the image. Shown overlaid on the first (left-hand) image are the epipolar line $\left[\mathbf{e}_{1}\right] \times \mathbf{p}_{1}$ and the perpendicular epipolar line $\left[\mathbf{e}_{1}\right]_{\times} \mathrm{I}\left[\mathbf{e}_{1}\right]_{\times} \mathbf{p}_{1}$. Overlaid on the second (right-hand) image are the corresponding epipolar lines $\mathrm{F} \mathbf{p}_{1}$ and $\mathrm{FI}\left[\mathbf{e}_{1}\right]_{\times} \mathbf{p}_{1}$. From these latter two lines one may compute the focal length $f_{2}$. Computation of the focal length $f_{2}$ will be very imprecise in this case, since the angle $\theta$ between these two lines is close to $90^{\circ}$, and also because one of the lines (actually $\mathrm{Fp}_{1}$ ) passes very close to the centre of the image, and hence (presumably) close to the principal point $\mathbf{p}_{2}$. Relative accuracy of the focal length $f_{2}$ is given by (3), and is proportional to the distance of $\mathbf{p}_{2}$ from each of these two epipolar lines, and also to the tangent of the angle $\theta$.

At the right is shown the focal length $f_{2}$ as a function of the principal point position $\mathbf{p}_{2}$. The focal length is represented by the intensity of the image. For a principal point in the black region of the image, the estimated value of $f_{2}^{2}$ is negative, meaning that the assumptions on position of the principal point are not viable (impossible situation).

Thus, $f_{2}^{2}=-A\left(\mathbf{p}_{1}, \mathbf{p}_{2}\right) B\left(\mathbf{p}_{1}, \mathbf{p}_{2}\right) / D_{2}(\mathbf{p} 1)$. Note that the estimated value of the focal length becomes zero where the numerator (that is, either $A$ or $B$ ) vanishes, and goes to infinity where the denominator $D$ vanishes. The value of $f_{2}^{2}$ will change sign on the union of the vanishing sets of $A, B$ and $D$. We look at each of these terms independently.

\subsection{Varying the Principal Point $\mathrm{p}_{2}$}

This situation has been explored in section 3 , The denominator $D\left(\mathbf{p}_{1}\right)$ does not depend on $\mathbf{p}_{2}$, and so is constant. We look at the vanishing sets of the two terms $A\left(\mathbf{p}_{1}, \mathbf{p}_{2}\right)$ and $B\left(\mathbf{p}_{1}, \mathbf{p}_{2}\right)$. As discussed above, $\mathrm{Fp}_{1}$ and $\mathrm{FI}\left[\mathbf{e}_{1}\right]_{\times} \mathbf{p}_{1}$ represent the epipolar lines in the second image, corresponding to the principal ray in the first image, and a ray perpendicular to it. When $\mathbf{p}_{2}$ lies on one of these epipolar lines, the numerator of (5) vanishes. Consequently, the zero set of $A\left(\mathbf{p}_{1}, \mathbf{p}_{2}\right) B\left(\mathbf{p}_{2}, \mathbf{p}_{2}\right)$ for fixed $\mathbf{p}_{1}$ and varying $\mathbf{p}_{2}$ consists of a pair of epipolar lines in the second image. The image plane is divided into four sections by these epipolar lines and the principal point $\mathbf{p}_{2}$ must lie in the acute-angled region of the plane. This is illustrated in Fig. 7 .

It may be noted that here and elsewhere (particularly in Fig. 9) the most probable principal point (image centre) sometimes lies in the dark part of the diagram, indicating an imaginary focal length. This is because when the principal ray passes near the centre of the image, the angle between the two lines dividing the image is necessarily close to $90^{\circ}$. If because of the instability of the computed fundamental matrix it falls on the 
wrong side of $90^{\circ}$, then the regions may swap sign, and lead to impossible values for the focal length. In these examples the fundamental matrix was computed using a Maximum Likelihood bundle-adjustment method, which should give optimal results - nevertheless this unfavourable situation can occur. A method that avoids this problem by placing $a$ priori conditions on the positions of the principal point and (optionally) the focal lengths was presented in [7]. It uses Bougnoux's formula to constrain the values of the focal length and principal points while finding a compatible fundamental matrix.

\subsection{Varying the Principal Point $\mathrm{p}_{1}$}

Next we consider the effect of varying the principal point $\mathbf{p}_{1}$ on the estimate of the focal length $f_{2}$. In this section we will assume that the principal point $\mathbf{p}_{2}$ of the second camera is known and fixed at a given value. As $\mathbf{p}_{1}$ varies, formula (5) give an estimate for the focal length $f_{2}$.

Vanishing set of $A\left(\mathbf{p}_{1}, \mathbf{p}_{2}\right) B\left(\mathbf{p}_{1}, \mathbf{p}_{2}\right)$. Since $\mathrm{F}$ is the fundamental matrix, $\mathrm{F}^{\top} \mathbf{p}_{2}$ is the epipolar line in the first image corresponding to the point $\mathbf{p}_{2}$. Thus the set of points $\mathbf{p}_{1}$ at which $B\left(\mathbf{p}_{1}, \mathbf{p}_{2}\right)=\mathbf{p}_{1}^{\top} \mathrm{F}^{\top} \mathbf{p}_{2}$ vanishes is precisely the set of points on the epipolar line $\mathrm{F}^{\top} \mathbf{p}_{2}$.

On the other hand, according to Proposition $3.1,\left[\mathbf{e}_{1}\right]_{\times} \mathrm{IF}^{\top} \mathbf{p}_{2}$ is the line perpendicular to $\mathrm{F}^{\top} \mathbf{p}_{2}$. Hence $A\left(\mathbf{p}_{1}, \mathbf{p}_{2}\right)=\mathbf{p}_{1}^{\top}\left[\mathbf{e}_{1}\right]_{\times} \mathrm{IF}^{\top} \mathbf{p}_{2}$ vanishes when $\mathbf{p}_{1}$ lies on the epipolar line perpendicular to $\mathrm{F}^{\top} \mathbf{p}_{2}$.

4.1. For a given value of $\mathbf{p}_{2}$, the set of points $\mathbf{p}_{1}$ such that the numerator $A\left(\mathbf{p}_{1}, \mathbf{p}_{2}\right) B\left(\mathbf{p}_{1}, \mathbf{p}_{2}\right)$ of (5) vanishes consists of the epipolar line $\mathrm{F}^{\top} \mathbf{p}_{2}$ and the epipolar line perpendicular to it.

Vanishing set of $D$. We now turn to the denominator of (5), namely $D\left(\mathbf{p}_{1}\right)=$ $\mathbf{p}_{1}^{\top}\left(\left[\mathbf{e}_{1}\right]_{\times} I F^{\top} I F\right) \mathbf{p}_{1}$. Note that this is independent of $\mathbf{p}_{2}$. From the form of this expression, the vanishing set is a conic. It will be shown that in fact it is a degenerate conic, consisting of two perpendicular lines through the epipole $\mathbf{e}_{1}$.

First of all one observes (by simple substitution and using the fact that $\mathrm{Fe}_{1}=0$ ) that the epipole $\mathbf{e}_{1}$ belongs to this vanishing set. If $\mathbf{p}_{1}$ is any point such that $D\left(\mathbf{p}_{1}\right)=0$, then $D\left(\mathbf{p}_{1}+\alpha \mathbf{e}_{1}\right)=0$ for any $\alpha$, and hence the vanishing set is made up of lines passing through $\mathbf{e}_{1}$, epipolar lines.

Now let $\mathbf{p}_{1}$ be a point at infinity, and let $\mathbf{p}_{1}^{\prime}$ be the point at infinity on the epipolar line normal to $\left[\mathbf{e}_{1}\right]_{\times} \mathbf{p}_{1}$. By symmetry, $\mathbf{p}_{1}^{\prime \prime}=\left(\mathbf{p}_{1}^{\prime}\right)^{\prime}=\mathbf{p}_{1}$. By Proposition $3.1 \mathbf{p}_{1}^{\prime}=$ $\mathrm{I}\left[\mathbf{e}_{1}\right]_{\times} \mathbf{p}_{1}$, and so $D\left(\mathbf{p}_{1}\right)=\mathbf{p}_{1}^{\prime \top}\left(\mathrm{F}^{\top} \mathrm{IF}\right) \mathbf{p}_{1}$. Substituting $\mathbf{p}_{1}^{\prime}$ for $\mathbf{p}_{1}$ in this expression gives

$$
D\left(\mathbf{p}_{1}^{\prime}\right)=\mathbf{p}_{1}^{\prime \prime \top}\left(\mathrm{F}^{\top} \mathrm{IF}\right) \mathbf{p}_{1}^{\prime}=\mathbf{p}_{1}^{\top}\left(\mathrm{F}^{\top} \mathrm{IF}\right) \mathbf{p}_{1}^{\prime}=\mathbf{p}_{1}^{\prime \top}\left(\mathrm{F}^{\top} \mathrm{IF}\right) \mathbf{p}_{1} \text { (by transposing) }=D\left(\mathbf{p}_{1}\right) .
$$

Thus, $D\left(\mathbf{p}_{1}^{\prime}\right)$ vanishes if and only if $D\left(\mathbf{p}_{1}\right)$ does. Consequently, the zero set of $D\left(\mathbf{p}_{1}\right)$ consists of a pair of orthogonal epipolar lines. Summarizing this complete discussion.

4.2. For a given value of $\mathbf{p}_{2}$, the set of points $\mathbf{p}_{1}$ for which the estimated value of $f_{2}^{2}$ changes sign consists of two pairs of orthogonal epipolar lines in image 1 . The value of $f_{2}^{2}$ tends to zero at one pair of lines, and to infinity at the other pair of lines.

This is illustrated in Fig. 8 


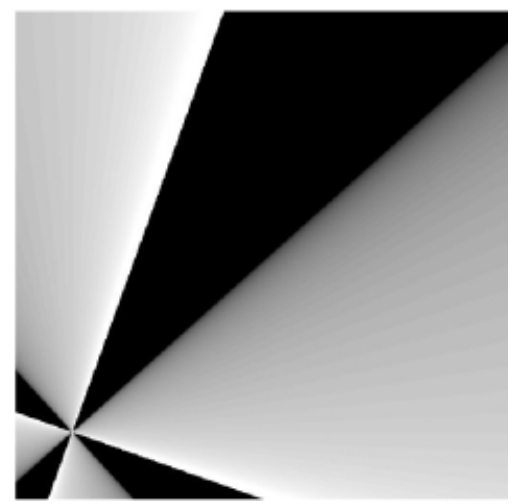

Fig. 8. Assuming $\mathbf{p}_{2}$ is fixed, and $\mathbf{p}_{1}$ is varying, the singular set for estimation of $f_{2}$ consists of points $\mathbf{p}_{1}$ lying on two pairs of orthogonal epipolar lines. The estimated value of $f_{2}$, according to (5) tends to 0 and $\infty$ as $\mathbf{p}_{1}$ approaches the alternating epipolar lines. The value of $f_{2}$ is only defined for $\mathbf{p}_{1}$ in alternating sectors of the image as shown, since $f_{2}^{2}$ is negative for $\mathbf{p}_{1}$ in the other (dark) sectors.

\section{Varying both Principal Points}

The previous sections have assumed that one of the principal points was fixed, and the other was allowed to vary. In some instances it may be more realistic to assume that the principal point is the same in both images, albeit not known exactly. Therefore, in this section, we assume that $\mathbf{p}_{1}=\mathbf{p}_{2}=\mathbf{p}$, and examine how the focal length estimate depends on the position of this common principal point. In this case, the formula for $f_{2}$ is as follows.

$$
f_{2}^{2}=-\frac{\left(\mathbf{p}^{\top}\left[\mathbf{e}_{1}\right]_{\times} \mathrm{IF} F^{\top} \mathbf{p}\right)\left(\mathbf{p}^{\top} F^{\top} \mathbf{p}\right)}{\mathbf{p}^{\top}\left(\left[\mathbf{e}_{1}\right]_{\times} \mathrm{IF}\right.}=-\frac{A(\mathbf{p}, \mathbf{p}) B(\mathbf{p}, \mathbf{p})}{D(\mathbf{p})} .
$$

We have seen already that the zero-set of $D(\mathbf{p})$ consists of a pair of orthogonal epipolar lines through the epipole $\mathbf{e}_{1}$. The zero sets of the expressions $A(\mathbf{p}, \mathbf{p})=\mathbf{p}^{\top} \mathrm{F}^{\top} \mathbf{p}$ and $B(\mathbf{p}, \mathbf{p})=\mathbf{p}^{\top}\left[\mathbf{e}_{1}\right]_{\times} \operatorname{IF}^{\top} \mathbf{p}$ are conics. It is easy to see that each of these expressions vanishes at both epipoles $\mathbf{e}_{1}$ and $\mathbf{e}_{2}$, hence the conics pass through the two epipoles. In addition, it may be verified that the tangents to these two conics at the epipole $\mathbf{e}_{1}$ are orthogonal. To see that, recall that the tangent at a point $\mathrm{x}$ on a conic defined by matrix $\mathrm{C}$ is the line $\left(\mathrm{C}+\mathrm{C}^{\top}\right) \mathbf{x}$. Applying this, one finds that the tangents to these two conics at the point $\mathbf{e}_{1}$ are $\left(\mathrm{F}^{\top}+\mathrm{F}\right) \mathbf{e}_{1}=\mathrm{F}^{\top} \mathbf{e}_{1}$ and $\left(\left[\mathbf{e}_{1}\right]_{\times} \mathrm{IF}{ }^{\top}-\mathrm{FI}\left[\mathbf{e}_{1}\right]_{\times}\right) \mathbf{e}_{1}=\left[\mathbf{e}_{1}\right]_{\times} \mathrm{IF}^{\top} \mathbf{e}_{1}$, which according to Proposition 3.1 are orthogonal lines through $\mathbf{e}_{1}$. Summarizing this gives the following result.

5.1. As the position of the principal point $\mathbf{p}_{1}=\mathbf{p}_{2}=\mathbf{p}$ varies, the singular set where the value of the focal length $f_{2}^{2}$ changes sign has the following components.

1. Two orthogonal epipolar lines passing through the epipole $\mathbf{e}_{1}$. As the assumed position of $\mathbf{p}$ approaches these lines, the value of $f_{2}^{2}$ tends to infinity. 

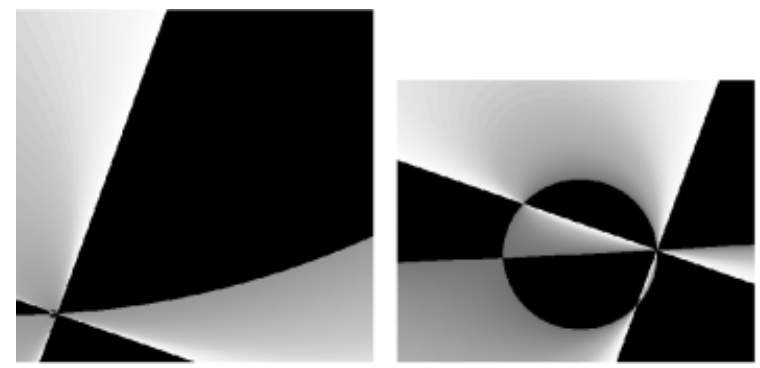

Fig. 9. Museum images: the geometry of the allowable positions of the principal point, assuming that it is the same in both images (shown at left). The plane is divided into regions by three conics (one of them degenerate). Black regions represent impossible locations for the principal point. Inside the allowable (white) regions, the focal length approaches infinity at the pair of orthogonal lines (degenerate conic), and zero at the non-degenerate conics. At right is an enlargement of the bottom left-hand corner, showing the conic corresponding to the denominator of (6).

2. Two conics passing through the two epipoles $\mathbf{e}_{1}$ and $\mathbf{e}_{2}$. The conics are orthogonal at $\mathbf{e}_{1}$. As $\mathbf{p}$ approaches these conics, the estimated value of $f_{1}^{2}$ tends to zero.

These lines divide the plane into regions. The value of $f_{2}^{2}$ is negative on alternate regions, which therefore represent impossible positions for the principal point.

For an illustration of this, see Fig. 9 .

\section{Reconstruction Accuracy}

Previous sections have demonstrated that the camera calibration, particularly the focal length, depends closely on the assumed position of the principal point. It has often been objected that this may be true, but it does not affect the reconstructed scene. This belief will be shown to be unsafe by an analysis of how angles in the reconstructed scene vary with the principal point and focal length estimates. The dependency of scene angles on calibration parameters is well known, and was noted in [2]. Here it will be shown that the effect can be quite marked, and in particular a simple method of visualizing the effect will be given.

A simple method of measuring some angles in a reconstructed scene utilizes a concept called the conformal point in an image. This concept was introduced in [6] where its application to visual odometry was demonstrated. The conformal point is defined relative to a plane of interest in the scene.

- The conformal point relative to any plane in the scene is located a distance $\left(f^{2}+d^{2}\right)^{1 / 2}$ from the vanishing line of the plane (the horizon), along a perpendicular to the horizon through the principal point. Here $f$ is the focal length of the camera, and $d$ is the distance in the image from the principal point to the horizon.

Note that the position of the conformal point is directly dependent on the focal length and the position of the principal point. Note that in Fig.2 the conformal point is located along the line hv a distance from $\mathbf{h}$ equal to the length of the chord $\mathbf{a h}$. 


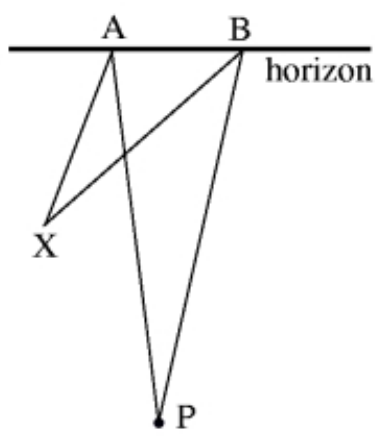

Fig. 10. To measure an angle between two lines in the image, the lines are extended to meet the horizon at points $A$ and $B$. The true angle between the two lines is equal to the angle $\angle A P B$, where $P$ is the conformal point. The position of the conformal point is explained in the text.

The interesting property of the conformal point is illustrated in Fig. 10, showing how it may be used to measure angles between lines in a plane. A proof of the correctness of this method is given in [6]. We apply this method of angle-measurement to the image shown in Fig. 3, and use it to estimate the angle between the door and the wall. According to the construction of Fig. 10, the required angle is the angle subtended at the conformal point by the vanishing points of the two directions under consideration. Note that in this example these two vanishing points are the two points (intersection of the green lines) used to construct the horizon line. The conformal point may be easily constructed assuming positions of the principal point of the image at the top, centre and bottom of the image along the vertical line. The estimated angles are

1. For principal point at the top of the image : $88^{\circ}$.

2. For principal point at the centre of the image $: 69^{\circ}$.

3. For principal point at the bottom of the image : $56^{\circ}$.

Thus in this example at least computed angles in the scene (properties of a Euclidean reconstruction based on a computed calibration) are very dependent on the assumed position of the principal point.

Beyond illustrating the variation of estimated scene geometry, this method gives a quick intuitive method of evaluating the degree of variation as a function of assumed principal point. This allows an operator to ascertain the effect of an incorrect placement of the principal point.

\section{Conclusion}

We have argued that the assumed position of the principal point may have a large effect on the estimated focal length of the cameras, and also the $3 \mathrm{D}$ reconstruction accuracy, for certain single-view and two-view calibration scenarios. The two-view calibration method analyzed here has been shown previously ([9]) to have degenerate configurations when the principal rays of the cameras meet. We have given here a simple analysis of how the 
quality of the focal length estimate degenerates when the principal ray of one camera lies close to the epipolar line corresponding to the other principal point. It is shown that in fact, the relative uncertainty in the the focal length estimated by this method is inversely proportional to the distance of the principal point to this epipolar line. Because of this sensitivity, the practicality of estimating focal lengths from two views is doubtful. However, in separately published work ([7]) we have proposed a method for mitigating some of the instabilities involved in estimating the principal point and focal length by assigning a priori bounds and variances for their values.

The method and analysis of this paper apply to other imaging scenarios, such as planar motion ([1] in which it is also possible to compute the image positions of the horizon and apex. A new interpretation of Bougnoux's focal length formula, is also given here, relating it to the geometry of the principal rays.

\section{References}

[1] M. Armstrong, A. Zisserman, and R. Hartley. Self-calibration from image triplets. In Proc. 4th European Conference on Computer Vision, Cambridge, LNCS 1064/5, pages 3-16. Springer-Verlag, 1996.

[2] S. Bougnoux. From Projective to Euclidean space under any practical situation, a criticism of self-calibration. In Proc. 6th International Conference on Computer Vision, Bombay, India, pages 790-796, January 1998.

[3] B. Caprile and V. Torre. Using vanishing points for camera calibration. International Journal of Computer Vision, 4:127-140, 1990.

[4] R. Cipolla, D. P. Robertson, and E. G. Boyer. Photobuilder - 3D models of architectural scenes from uncalibrated images. In Proc. IEEE International Conference on Multimedia Computing and Systems, volume I, pages 25 - 31, June 1999.

[5] R. I. Hartley. Estimation of relative camera positions for uncalibrated cameras. In Proc. 2nd European Conference on Computer Vision, Santa Margharita Ligure, Italy, LNCS 588, pages 579-587. Springer-Verlag, 1992.

[6] R. I. Hartley and C Silpa-Anan. Visual navigation in a plane using the conformal point. In International Symposium on Robotics Research, pages to appear - available at http://www.anu.edu.au/ hartley, 2001.

[7] R. I. Hartley and C Silpa-Anan. Reconstruction from two views using approximate calibration. In $A C C V$, pages 338-343, 2002.

[8] R. I. Hartley and A. Zisserman. Multiple View Geometry in Computer Vision. Cambridge University Press, 2000.

[9] G. Newsam, D. Q. Huynh, M. Brooks, and H. P. Pan. Recovering unknown focal lengths in self-calibration: An essentially linear algorithm and degenerate configurations. In Int. Arch. Photogrammetry \& Remote Sensing, volume XXXI-B3, pages 575-80, Vienna, 1996.

[10] M. Pollefeys, R. Koch, and L. Van Gool. Self calibration and metric reconstruction in spite of varying and unknown internal camera parameters. In Proc. 6th International Conference on Computer Vision, Bombay, India, pages 90-96, 1998. 